\title{
Vorschlag zur bodenartlichen Kennzeichnung von Wattsedimenten ${ }^{1}$ )
}

\author{
Von J. L. Ruy'rer, Hannover \\ Mit 4 Abbildungen im Text und 1 Tabelle
}

$\mathrm{Zusammenf}$ assung. Im Rahmen einer Spezialkartierung im ostfriesischen Watt ergab sich die Frage nach einer zweckmäßigen Einteilung und kartenmäßigen Darstellung der dort auftretenden Bodenarten (Korngrößengemische). Die hier vorgeschlagene Gruppierung sollte weitgehend den chemisch-physikalischen Eigenschaften dieser rezenten Wattsedimente entsprechen und mit der Bodenarten-Ansprache der Marschkartierung des Niedersächsischen Landesamtes für Bodenforschung übereinstimmen. Anhand der durchgeführten Bodenuntersuchungen werden die wichtigsten Eigenschaften der angetroffenen Wattsedimente und ihre Beziehung zueinander besprochen und die Grenzwerte für die vorgenommene Gliederung dargelegt.

$\mathrm{S} \mathrm{u} \mathrm{m} \mathrm{m}$ a r y. During a special mapping in the tidal flat of Eastern Frisia the question arose as to how the soils in this area could most suitably be classified and represented on maps (grain size mixtures). The grouping suggested in this paper is done according to chemical and physical properties of these recent tidal flat sediments. Simultaneously conformity with the soil definitions of the marsh mapping by the "Niedersächsisches Landesamt für Bodenforschung" was tried to be reached. Based on the soil analysis, the most outstanding qualities of tidal flat sediments found here and their relations are being discussed. Furthermore the boundary values for the classification are investigated.

Im Sommer 1959 wurde im Watt der Leybucht eine geologisch-bodenkundliche Versuchskartierung als Gemeinschaftsarbeit des Niedersächsischen Landesamtes für Bodenforschung mit der Forschungsstelle Norderney durchgeführt. Es sollte geprüft werden, inwieweit das inzwischen auf dem Festland crarbeitete Verfahren der Marschkartierung (Geologisch-bodenkundliche Karte der niedersächs. Marschen 1:5000, 1956-1960) im Watt technisch durchführbar ist und welche sinnvolle Anwendung für die Fragen der Landgewinnung und der künftigen Nutzung neu einzudeichender Wattflächen sich daraus ergibt. Darüberhinaus sollte durch die hiermit verbundenen analytischen Feststellungen (Methoden: siehe Beihefte zu den geolog.-bodenkundl. Karten 1:5000) ein Beitrag zu dem Problem der chemikalisch-physikalischen Eigenschaften von rezenten Wattsedimenten geleistet werden.

Die größte Schwierigkeit für die Erstellung einer Wattkarte lag in dem Fehlen einer verbindlichen Nomenklatur für die Bezeichnung der im Watt vorkommenden Korngrößengemische. Diese Tatsache erschwert z. Zt. die Aufstellung einer allgemein verständlichen und über das Gebiet der Leybucht hinaus brauchbaren Legende bezüglich der Charakterisierung der hier auftretenden Korngrößengemische und ihrer kartenmäßigen Darstellung.

Die bisherigen Gliederungen (Wohlenberg 1937, Linke 1937, Plath 1943, C. D. MüLler 1957, 1958) konnten im Rahmen des o. a. Kartiervorhabens nicht voll befriedigen. Diese Gliederungen waren entweder zu grob oder zu unübersichtlich. Weiterhin konnte keine exakte Abgrenzung der einzelnen Bodenartengruppen vorgenommen werden, oder einer Abgrenzung lagen ungleiche Fraktionen zugrunde. Darüberhinaus wurden Schlämmanalysenmethoden verwendet, die keinen Vergleich mit den heute üblichen Verfahren zulassen und fast immer den Tonanteil aufgrund zu geringer Dispergierung vernachlässigten.

Es liegt auf der Hand, daß die Korngrößenzusammensetzung in einem hinsichtlich der

1) Auszug aus einem Vortrag, gehalten am 9. 6. 1960 anläßlich der 27. Tagung der Arbeitsgemeinschaft Nordwestdeutscher Geologen, Norderney 8.-11. 6. 1960. 


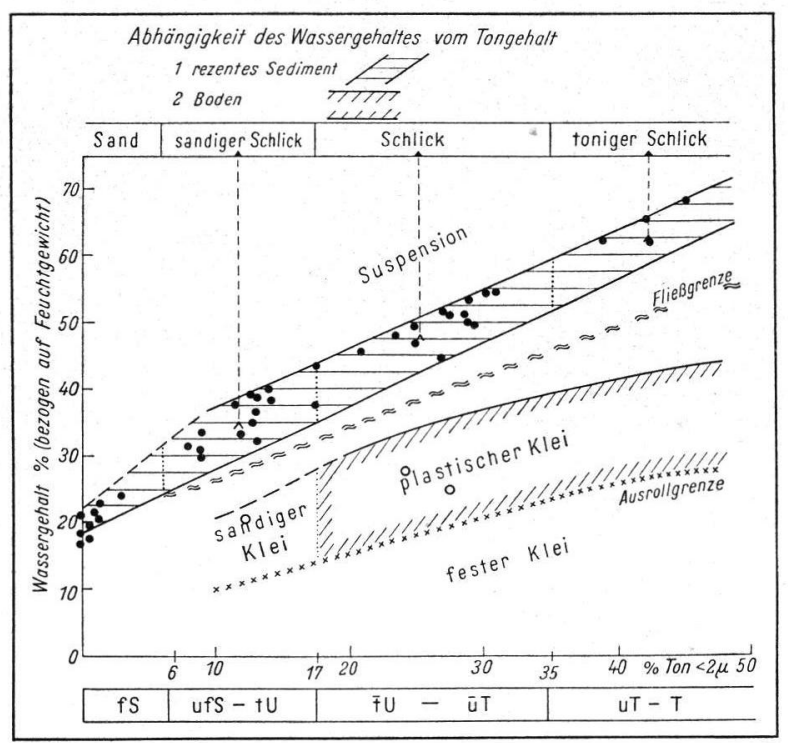

Abb. 1. Wassergehalt und Konsistenz. - $\mathrm{H}_{2} \mathrm{O}-\mathrm{G}$-halte von rezenten Sedimenten; $\mathrm{O} \mathrm{H}_{2} \mathrm{O}-\mathrm{Gehalte}$ von überschlickten fossilen Bodenbildungshorizonten.

Salinität gleichartigen Sedimentationsraum von besonderer Bedeutung ist. Dennoch kann die Korngrößenzusammensetzung nur dann als Kriterium für die Einteilung der Wattsedimente dienen, wenn andere wichtige Eigenschaften hiermit korrelieren.

Eine wichtige Eigenschaft von Wattsedimenten ist ihr Wassergehalt. Es wurde untersucht, welche Zusammenhänge zwischen den Tongehalten $(\langle 2 \mu)$, die mit dem angewendeten Analysenverfahren (Jung-LüTtMeR 1955 - nach vorheriger hin reichender Entsalzung und Humuszerstörung mit $-\mathrm{H}_{2} \mathrm{O}_{2}$ ) ermittelt wurden, und den $\mathrm{H}_{2} \mathrm{O}$-Gehalten rezenter Sedimente bestehen. Zusätzlich wurden die Wassergehalte von bereits mehr oder weniger vorentwässerten Substraten (Böden) innerhalb der Fließ- und Ausrollgrenze, bezogen auf den Tongehalt, dargestellt. Das Ergebnis zeigt Abb. 1.

Es ergibt sich aus der Abbildung, daß eine enge Beziehung zwischen Ton- und $\mathrm{H}_{2} \mathrm{O}$ Gehalt für junge Sedimente besteht. Daraus folgt, daß rezenten Sedimenten je nach Tongehalt bestimmte $\mathrm{H}_{2} \mathrm{O}-\mathrm{Gehalte}$ bzw. je nach $\mathrm{H}_{2} \mathrm{O}$-Gehalt bestimmte Tongehalte zugeordnet werden können. Wie die Abbildung 1 zeigt, gilt dies nicht für die o. a. vorentwässerten Böden (Klei). Mit anderen Worten: Ein rezentes Sediment hat den Wassergehalt, der seiner Korngrößenzusammensetzung entspricht. Damit ist gleichzeitig eine Aussage über die jeweilige Lagerungsdichte bzw. das Porenvolumen eines jungen Sedimentes möglich, da die tidebedingte täglich zweimal erfolgende Überstauung einen nennenswerten lufterfüllten Porenraum zunächst nicht zuläßt. Diese hier diskutierte Beziehung gilt nur dann nicht, wenn ein abnormer Gehalt an organischer Substanz vorliegt.

In der Abbildung $2 a$ sind die gefundenen Abhängigkeiten zwischen Tongehalt und Prozent-Anteilen an organischer Substanz und $\mathrm{CaCO}_{3}$ aufgezeigt. Diese Beziehung gilt als Regelfall für den hier untersuchten Raum, wie dies ebenfalls bereits von anderen Autoren festgestellt wurde (Linke 1937, Plath 1943, Pons \& Wiggers 1959, 1960).

Abbildung $2 \mathrm{~b}$ zeigt die Abhängigkeit zwischen dem Tongehalt $(\langle 2 \mu)$ und dem T-Wert (Sorptionskapazität = Kationenaustauschkapazität) als auch dem quantitativen Gehalt an freien Salzen. Die letztere Beziehung ist wiederum abhängig von der Salinität des Sedimentationsraumes und in diesem Fall mit 25\% zugrunde gelegt. 


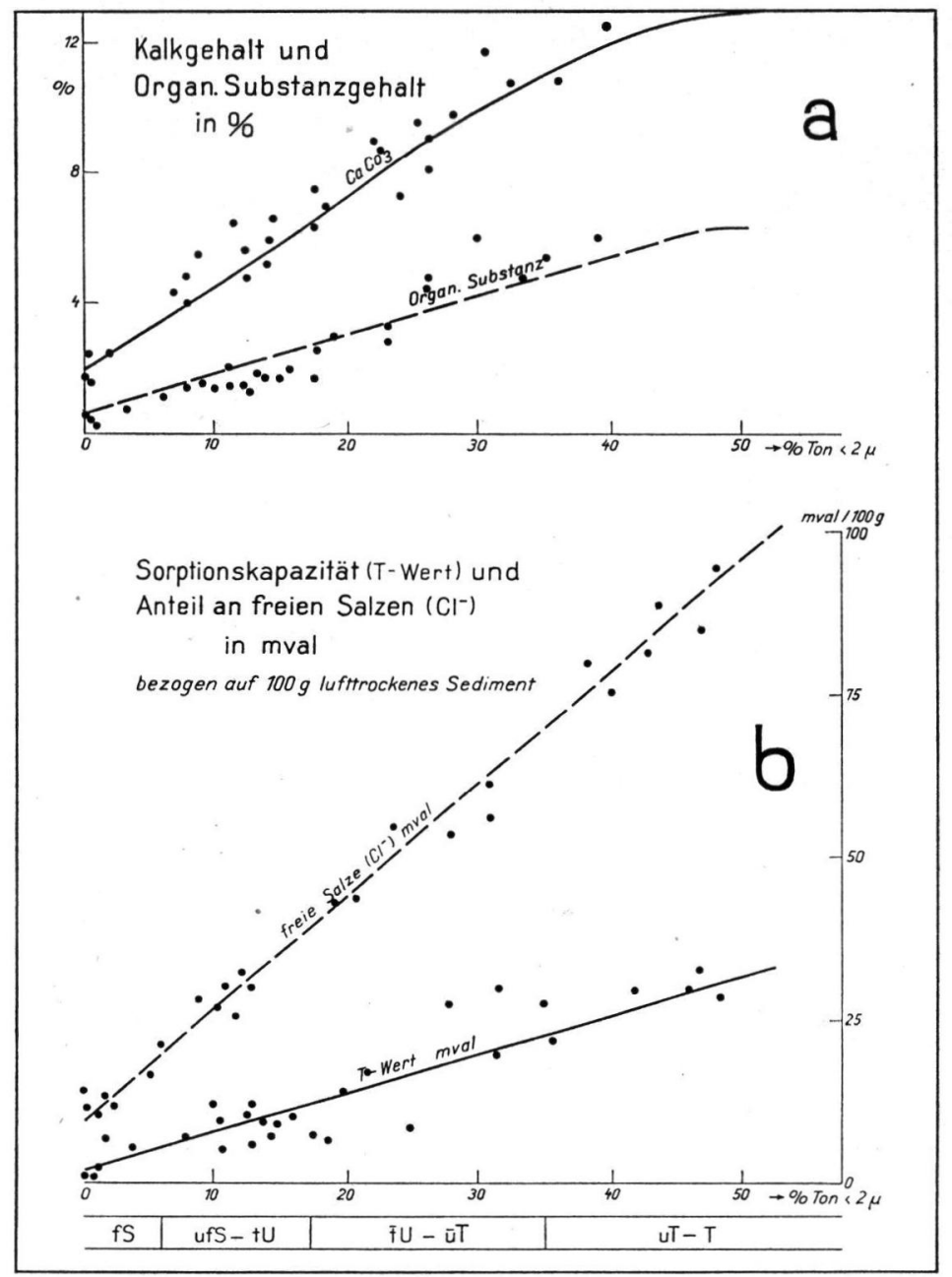

Abb. 2. Eigenschaften rezenter Sedimente in Abhängigkeit vom Tongehalt.

In der Abbildung 3 ist die Beziehung zwischen dem $\mathrm{H}_{z} \mathrm{O}-$ Gehalt (bezogen auf Frischgewicht) und dem Salzgehalt des Bodenwassers in mval Cl-/Ltr. aufgetragen. Der Vorteil dieser Darstellung liegt in der Klarstellung der Zusammenhänge zwischen $\mathrm{H}_{2} \mathrm{O}$-Gehalt des Korngrößengemisches - und damit bei rezenten Sedimenten mit dem Tongehalt der Salinität des Sedimentationsraumes und dem möglichen Anteil an freien Salzen in mval $\mathrm{Cl}^{-}$, bezogen auf $100 \mathrm{~g}$ lufttrockene Substanz.

Es ergibt sich somit, daß Abhängigkeiten bestehen zwischen dem Tongehalt, $\mathrm{H}_{2} \mathrm{O}$ Gehalt, dem Gehalt an freien Salzen und der Salinität des Sedimentationsraumes. Wenn eine dieser 4 Größen nicht bekannt ist, kann sie somit ohne weiteres überschlägig mit Hilfe der Abbildungen 1 und 3 abgeleitet werden.

Diese Darlegungen beruhen auf der eingehenden Untersuchung von etwa 70 Bodenproben. Sie bedeuten ein vorläufiges Ergebnis der unter einer speziellen Fragestellung angestellten Überlegungen, die durch weitere Untersuchungen erweitert und vertieft werden sollen. 


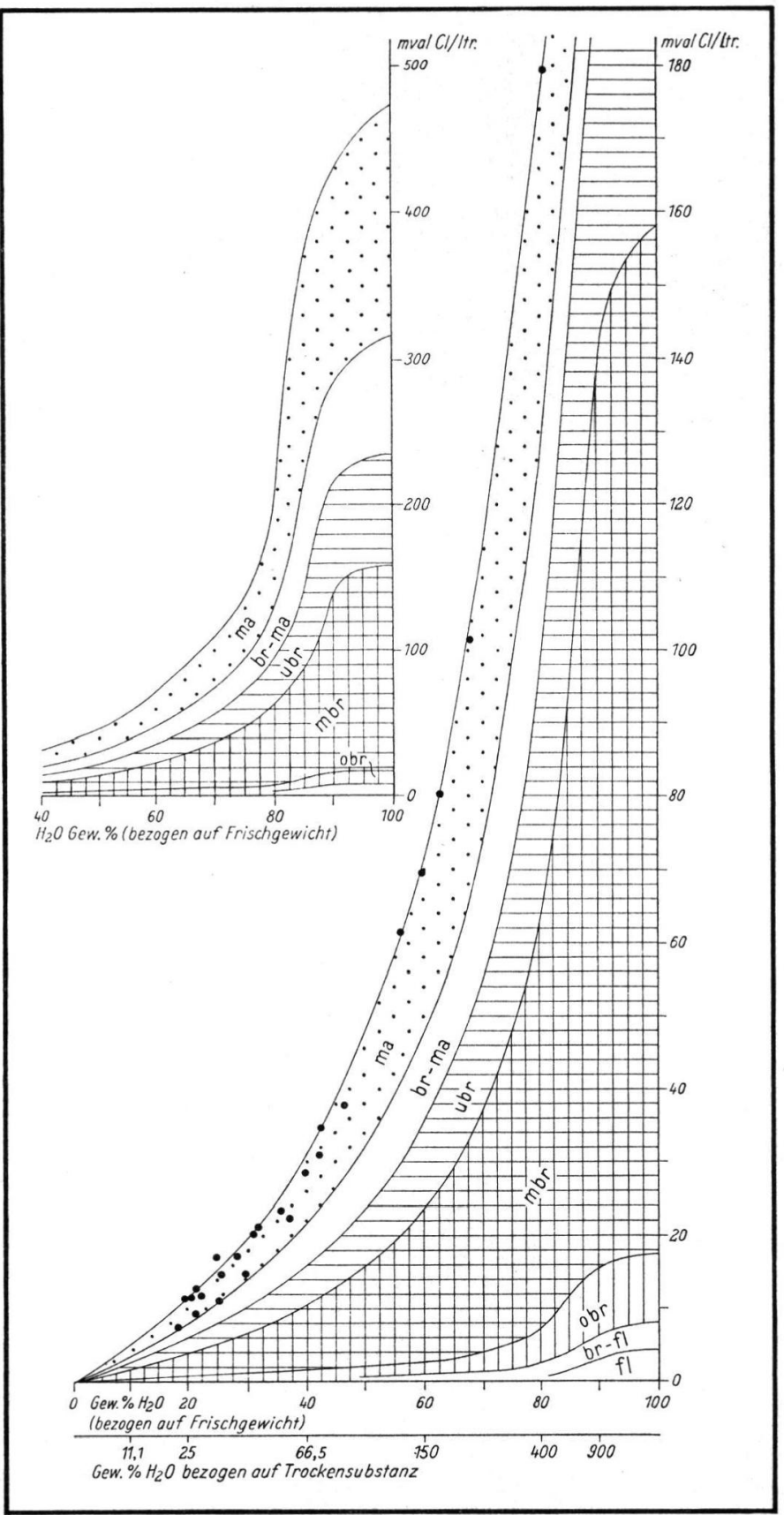

Abb. 3. Beziehung zwischen Salzgehalt, Bodenfeuchte und Salinität des Sedimentationsraumes. $\mathrm{ma}=$ marin, $\mathrm{br}-\mathrm{ma}=$ brackisch-marin, $\mathrm{ubr}=$ unteres Brackwasser, $\mathrm{mbr}=$ mittl. Brackwasser, $\mathrm{obr}=$ oberes Brackwasser, $\mathrm{br}-\mathrm{fl}=$ brackisch-fluviatil, $\mathrm{fl}=$ fluviatil. Grundlagen dieser Abgrenzung: siehe Beiheft zur geo'ogisch-bodenkundl. Karte 1:5000. 


\begin{tabular}{|c|c|c|c|c|c|c|c|c|c|c|c|}
\hline \multirow{2}{*}{$\begin{array}{c}* \\
\text { Boden-1 } \\
\text { arten }\end{array}$} & \multirow{2}{*}{$\begin{array}{c}\text { Boden- } \\
\text { arten- } \\
\text { gruppen }\end{array}$} & \multirow{2}{*}{\multicolumn{2}{|c|}{$\begin{array}{c}\text { Zusammenfossende Bezeichng. } \\
\text { von } \\
\text { Korngrößengemischen } \\
\text { in } \\
\text { unentwässertem Zustand } \\
\end{array}$}} & \multicolumn{3}{|c|}{ Grenzwerte } & \multicolumn{3}{|c|}{ bezüglich } & \multirow{2}{*}{\multicolumn{2}{|c|}{$\begin{array}{l}\text { Bezeichnung u Unterteilung } \\
\text { von } \\
\text { Wattflächen nach den an } \\
\text { ihrer Oberflöche ouftretenden } \\
\text { KorngröBengemischen }\end{array}$}} \\
\hline & & & & \multirow{3}{*}{$\begin{array}{c}\text { Ton } \\
<2 \mu \\
\%\end{array}$} & \multirow{2}{*}{\begin{tabular}{c|} 
Feinsand \\
$>60 \mu$ \\
$\%$
\end{tabular} \mid} & \multirow{2}{*}{$\begin{array}{c}\mathrm{H}_{2} \mathrm{O} \text { Gehalt } \\
\begin{array}{c}\text { Bodent+H } \\
\%\end{array} \\
52-70\end{array}$} & \begin{tabular}{|c|} 
freie Salze \\
$\mathrm{Cl}^{-}$ \\
$\mathrm{m} \mathrm{val}$
\end{tabular} & $\begin{array}{r}T-\text { Wert } \\
\text { m val } \\
\end{array}$ & $\begin{array}{l}\text { Organ. } \\
\text { Subst. } \\
\%\end{array}$ & & \\
\hline \begin{tabular}{|l|} 
\\
$u^{\prime} T$ \\
\end{tabular} & Tone & toniger & & & & & $60-100$ & $>25$ & $6(>6)$ & toniges & \\
\hline UT & schluffige & Schlick & & & -10 & & $00-100$ & 200 & $01>01$ & Schlickwatt & \\
\hline$\overline{\mathbf{u} T}$ & Tone & & & & & & & & & & Schlickwatt \\
\hline $\begin{array}{r}\bar{f} U \\
\overline{\mathrm{f} f s} U \\
\end{array}$ & & Schlick & & $17-35$ & $20-40$ & $35-55$ & $40-60$ & $12-25$ & $4-6$ & Schlickwatt & \\
\hline$\frac{t U}{t f s U}$ & & schluffiger & & & & & & & & schluffiges & \\
\hline$t^{\prime} U$ & Schlutfe & Schlick & & $12-17$ & $20-60$ & $28-42$ & $30-40$ & & & & sandiges \\
\hline U & & & & & & & & $6-12$ & $1-4$ & 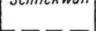 & \\
\hline \begin{tabular}{|l|}
$f^{\prime} u f S$ \\
$f S U$ \\
$f S^{\prime} U$ \\
$u f S$ \\
\end{tabular} & $\begin{array}{l}\text { Schluffe } \\
u . \\
\text { feinsand. } \\
\text { Schluffe } \\
\end{array}$ & $\begin{array}{l}\text { sandiger } \\
\text { Schlick }\end{array}$ & & $6-12$ & $60-85$ & $25-35$ & $15-30$ & & & $\begin{array}{l}\text { sandiges } \\
\text { schlickwatt }\end{array}$ & Schlickwatt \\
\hline fS & Feinsand & Feinsand & $\begin{array}{l}{ }_{0}^{\circ} \text { (watt) } \\
\circ \text { sand } \\
0 \\
0\end{array}$ & $<6$ & $>85$ & $18-25$ & $8-15$ & $2-6$ & $<1$ & Sandwatt & Sandwatt \\
\hline
\end{tabular}

Tabelle 1

Gruppierung und Grenzwerte der Wattsedimente in Verbindung mit der Bodenarten-Einteilung der Marschkartierung.

* Bei der Marschenkartierung des Niedersächsischen Landesamtes f. Bodenforschung seit 1959 übliche Einteilung.

** Die hier angegebenen Grenzwerte beziehen sich auf die Salinitätsverhältnisse der Leybucht $(25-30 \%$. Bei geringeren Salzgehalten sind entsprechende Werte aus Abb. 3 einzusetzen.

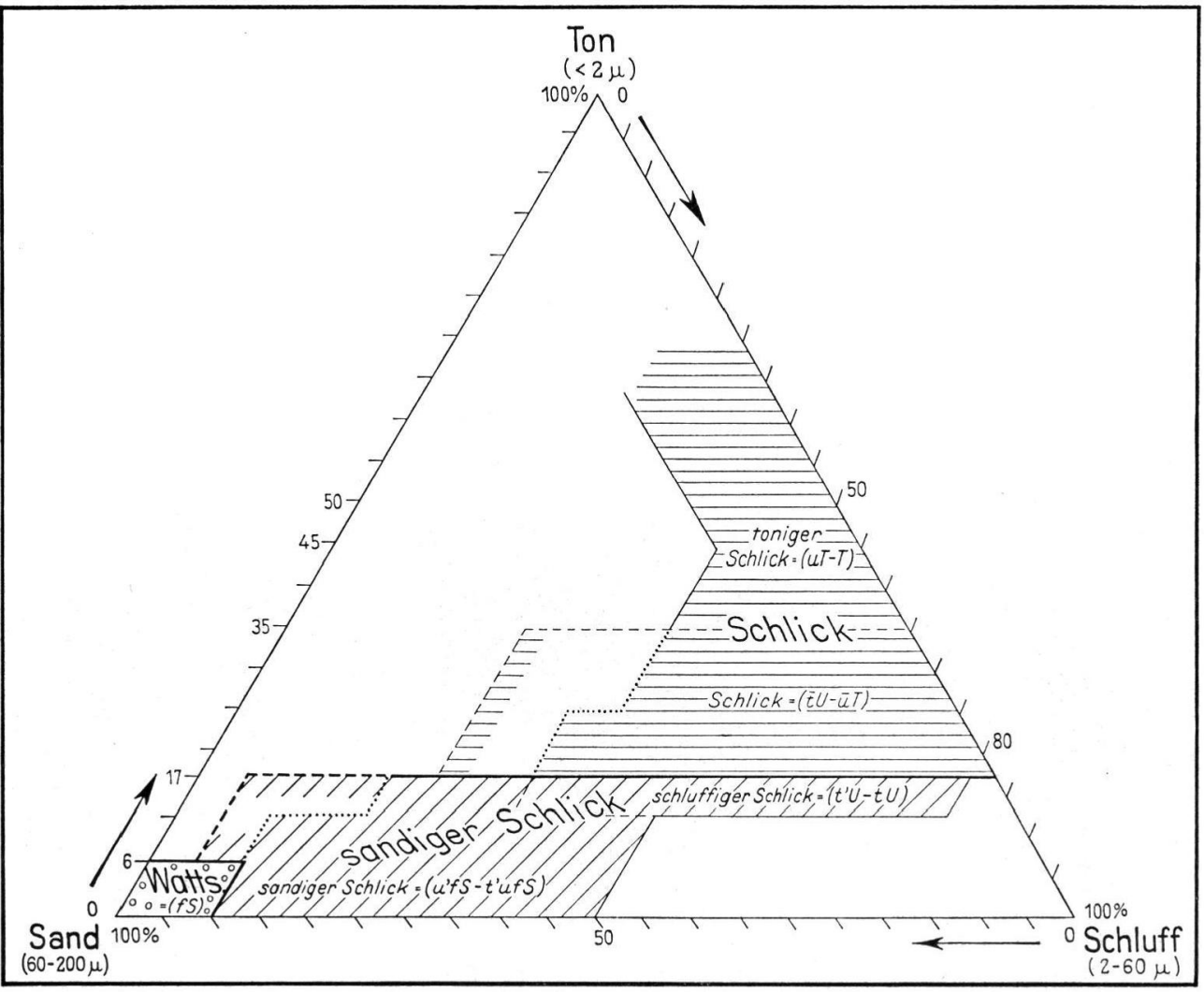

Abb. 4. Vorschlag zur Bezeichnung der Wattsedimente. 
Welche Schlußfolgerungen ergeben sich nun nach der eingangs gestellten Frage einer zweckmäßigen Gliederung dieser Wattsedimente?

Die bekannte Salinătät des untersuchten Wattengebietes ist relativ gleichmäßig (ca. 25\% Gleiche gilt für den $\mathrm{H}_{2} \mathrm{O}$-Gehalt und die damit verbundenen mechanischen Eigenschaften dieser Substrate, u. a. mögliche Setzungen, auf die in einer späteren Arbeit näher eingegangen werden soll.

Die Korngrößenzusammensetzung, insbesondere die genaue, durch a u s e ichende Dispergierung gewonnene Kenntnis des Tongehaltes $(\langle 2 \mu)$ wurde daher als besonders wichtiges Kriterium für die Kennzeichnung der Wattsedimente herangezogen.

Weiterhin wurde Wert darauf gelegt, die Korngrößenabstufung mit denen der Marschböden in Übereinstimmung zu bringen, weil dies hinsichtlich der Abschätzung der Eigenschaften bei einer eventuell späteren Bodennutzung von besonderer Bedeutung erschien. Die aufgrund dieser Überlegungen gewählte Einteilung ist aus Tabelle 1 und Abbildung 4 ersichtlich.

In der Tabelle 1 sind darüberhinaus überschlägig die den Korngrößengemischen zugehörigen Gehalte an $\mathrm{H}_{2} \mathrm{O}$, freien Salzen, T-Werten und Gehalten an organischer Substanz aufgeführt. Aus dieser Tabelle ergeben sich ebenfalls Vorschläge für eine definierte Bezeichnung von Wattflächen.

In Abbildung 4 sind ausschließlich die für die Wattsedimente vorgeschlagenen Bezeichnungen in einem Dreiecks-Schaubild dargestellt (bezüglich der Darstellungsform siehe SCHIRMER 1959).

Hierbei wurden die zahlreichen Korngrößenanalysen aus den ostfriesischen Wattgebieten von Herrn Dr. SINDOwSKI mitverwertet, die dieser dankenswerterweise zur Verfügung gestellt hat.

\section{Literatur}

Jung, L., \& LütTMER, J.: Über die Eignung des Natriumpyrophosphates zur Dispergierung bei der mechanischen Bodenanalyse. - Notizbl. hess. Landesamtes f. Bodenf. 83, S. 282-291, 1955.

Linke, O.: Die Biota des Jadebusenwattes. - Helgoländ. wiss. Meeresuntersuchung 1, S. 201-348, 1937.

MüLleR, C. D.: Biologisch-sedimentologische Untersuchung zur Frage des Uferabbruches beiderseits des Cappeler Tiefs. - Forschungsstelle Norderney, Jahresbericht 9, S. 117-130, 1957. - - Biologische und sedimentclogische Wattuntersuchung zwischen Westeraccumersiel und Neuharlingersiel an der ostfries. Küste. - Ebendort 10, S. 61-86, 1958.

Nieders. Landesamt für Bodenforschung, Hannover: Geologisch-bodenkundliche Karte der niedersächsischen Marschen 1:5000 und Beihefte. - Herausgegeben vom Niedersächs. Landesamt f. Bodenf., Hannover 1956-1960.

PLATh, M.: Die biologische Bestandsaufnahme als Verfahren zur Kennzeichnung der Wattsedimente und die Kartierung der nordfriesischen Watten. - Westküste, Kriegsheft 1943, S. 7-45.

Pons, L. J., \& Wiggers, A. J.: De holocene wordingsgeschiedenis van Noordholland en het Zuiderzeegebied. - Verh. k. nederl. aardrijksk. Genootschap 76, S. 104-152, 1959, 77, S. 3-57, 1959.

Schirmer, M.: Vorschlag für eine Dreieckdarstellung der Bodenarten in der Kulturtechnik. - Der Kulturtechniker 47, S. 197-208, 1959.

Wohlenberg, E.: Die Wattenmeerlebensgemeinschaften im Königshafen von Sylt. - Helgoländ. wiss. Meeresuntersuchg. 1, S. 1-92, 1937.

Manuskr. eingeg. 3. 9. 1960.

Anschrift des Verf.: J. L. Ruyter, Dipl.-Landwirt, Niedersächsisches Landesamt für Bodenforschung, Hannover, Wiesenstraße 1. 